\title{
El deseo de acceso y equidad en la educación matemática*
}

The Desire for Access and Equity in Mathematics Education

O desejo de acesso e equidade na educação matemática

\author{
Paola Valero** (iD o000-0002-5736-7562
}

\begin{abstract}
Artículo de revisión
Revista Colombiana de Educación, N.⒎ 73. Segundo semestre de 2017, Bogotá, Colombia.

doi: $10.17227 / 01203916.73$ rce 97.126

Para citar este artículo: Valero, P. (2017). El deseo de acceso y equidad en la educación matemática. Revista Colombiana de Educación, (73), 99-128.
\end{abstract}

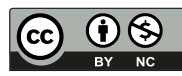

Recibido: 18/12/2016

Evaluado: 17/04/2017

* Este artículo desarrolla en profundidad las ideas planteadas en P. Valero y A. Arvola-Orlander, "Democracy and justice in math and science curriculum", que se publicará en el libro virtual de referencia Oxford Encyclopedia of Education. Este artículo es parte de la investigación del Centro Nórdico de Excelencia en Investigación Educativa "JustEd".

** Doctora en Educación Matemática. Profesora titular del Departamento de Educación Matemática y Científica, Universidad de Estocolmo, Suecia. Correo electrónico: paola.valero@mnd.su.se 


\title{
Resumen
}

La idea de que el fracaso en las matemáticas es un problema para la democracia y la equidad debe entenderse como parte de una configuración histórica donde el logro de los individuos y la población se conecta con el desarrollo de capital humano y el progreso social y económico. El deseo de una población con más capacidades matemáticas va de la mano de la generación de mecanismos de ordenación y clasificación de la población y la in(ex)clusión con relación al éxito/fracaso en las matemáticas escolares. La investigación internacional en educación matemática ha abordado este problema desde distintas posiciones teóricas. Dos de ellas se identifican: la posición del empoderamiento supone que las matemáticas transfieren sus atributos de poder a quienes las aprenden, o bien por sus características intrínsecas, o por sus aplicaciones, o por su carácter crítico. La posición de la desigualdad supone que las matemáticas como formas de conocimiento en sí o como parte de las prácticas escolares se entrelazan con mecanismos de clasificación social de acuerdo con otras categorías, como la habilidad, el género, el lenguaje, etc. Las tensiones que emergen en el campo de la educación matemática por la co-existencia de esta diversidad de visiones llama a tomar una posición ética y política sobre qué posibilidades hay para la investigación y la práctica.

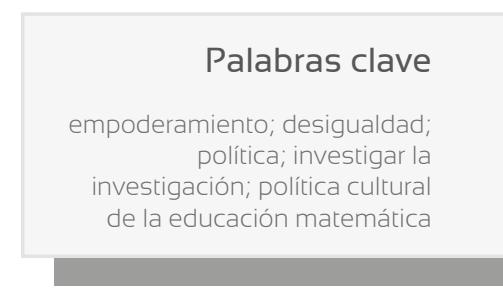

\section{Keywords}

empowerment; inequality; politics; researching research; cultural policy of mathematics education

\begin{abstract}
The idea that failure in mathematics is a problem for democracy and equity should be understood as a part of history where the achievements of individuals and the population is linked to the development of human capital, as well as social and economic progress. The desire for a population with higher math skills goes hand in hand with the generation of mechanisms to order and classify the population and the in(ex)clusion regarding success/failure in school mathematics. International research in math education has approached this issue from different theories, two of which are identified: the position of empowerment supposes that mathematics transfers its attributes of power to those who learn it, either because of its intrinsic characteristics, its applications, or its critical nature. The position of inequality supposes that mathematics, as forms of knowledge per se or as part of the school practices intertwined with mechanisms of social classification according to other categories, such as skill, gender, language, etc. Tensions emerging in the field of mathematics education due to the co-existence of these different views calls for taking an ethical and political position on the possibilities for research and practice.
\end{abstract}

\section{Resumo}

A ideia de que o fracasso nas matemáticas e um problema para a democracia a equidade deve ser entendido como parte de uma configuração histórica onde o sucesso dos indivíduos e a população está ligado ao desenvolvimento do capital humano e o progresso social e econômico. O desejo de uma população com mais capacidades matemáticas está unido à geração de mecanismos de ordenação e classificação da população e a in(ex)clução com relação ao sucesso/fracasso nas matemáticas escolares. A pesquisa internacional em educação matemática estudou esse problema desde diversas aborda. gens teóricas. Duas destas podem ser identificadas: a posição do empoderamento supõe que as matemáticas transferem seus atributos de poder para os que as aprendem, ou tal vez por suas caraterísticas intrínsecas, ou por suas aplicações, o pelo seu caráter crítico. A posição da desigualdade supõe que as matemáticas como formas de conhecimento em sim ou como parte das práticas escolares são vinculadas, como a habilidade, o gênero, a linguagem, etc. As tensões que emergem no campo da educação matemática pela coexistência dessa diversidade de visões convidam para adotar uma posição ética e política sobre que possibilidades existem para a investigação e a prática.

\section{Palavras chave}

empoderamento; desigualdade; política; pesquisar a pesquisa política cultural da educação matemática 


\section{Introducción}

Las matemáticas son vistas hoy en día una como una de las áreas centrales del currículo escolar pues, junto con la lengua materna y las ciencias, son consideradas como las materias que ofrecen conocimientos, habilidades y competencias clave para la participación en la cultura tecnológica contemporánea, en las actividades productivas del mundo laboral, y en procesos políticos democráticos. Esta visión no solo es parte de las justificaciones de matemáticos y educadores matemáticos sobre la importancia de su área. También hace parte importante de las políticas nacionales e internacionales en educación en varios lugares del mundo. En un país como Colombia esto se ve claramente en las discusiones alrededor de la importancia de mejorar los resultados de los estudiantes en pruebas nacionales e internacionales -como las pruebas PISA- y en esfuerzos por elevar masivamente las cualificaciones de los maestros de matemáticas, tanto por parte del Ministerio de Educación Nacional, como por algunos Gobiernos regionales. Más aún, tal interés no se restringe a aquellos involucrados en la educación, sino que también llega a asociaciones profesionales y empresariales en distintas partes del mundo, que promueven iniciativas para atraer a más jóvenes a las áreas de las matemáticas, ciencia, ingeniería y tecnología como una estrategia de competitividad en la sociedad del conocimiento globalizada. Simultáneamente una serie -también creciente- de mediciones de rendimiento en matemáticas en los ámbitos local, nacional e internacional han permitido constatar que hay distintos grupos y un gran número de personas que ni siquiera alcanzar los logros mínimos esperados. El fracaso escolar en matemáticas se ha vuelto persistente, y con él la potencial exclusión de muchos estudiantes de unirse al deseo por las matemáticas. ¡Es como si el deseo de expandir el alcance de más matemáticas para todos generara mecanismos de selección y exclusión de muchos!

El fracaso escolar en matemáticas no siempre ha sido un tema de preocupación como un asunto de equidad o de democracia. Por ejemplo, consideremos la idea que existe en muchos cursos de matemáticas de que solo muy pocos pueden "pasar" o ganar buenas calificaciones. Es más, en algunos casos un "buen profesor" es aquel tan bueno que solo los más inteligentes pueden "pasar" -o la mayoría debe reprobar-. Desde esta visión, la distribución de habilidad se comporta de manera "normal". Si esta es la situación "natural", ¿cuál es el problema con el fracaso? Este tipo de visión es todavía muy frecuente en muchos ámbitos educativos. Solo recientemente el fracaso escolar en matemáticas se ha convertido en un problema tanto social como de investigación. Primero, implícitamente la intención del estudio científico de la educación matemática ha sido abordar el fracaso a través de la mejora de la enseñanza y el aprendizaje 
con conocimiento experto. Pero desde hace poco tiempo que el fracaso escolar se convirtió en un problema de investigación, por ser un reto a la posibilidad democrática de acceso a los beneficios de la sociedad.

Mi intención en esta revisión de la literatura es mostrar cómo el creciente deseo por las matemáticas "para todos" va de la mano de la generación de ordenamientos y clasificaciones que generan cierre de acceso "para algunos". Esta idea es importante de entender en cualquier discusión sobre la educación matemática y la equidad. Comienzo por una breve historización del deseo por hacer de la educación matemática un elemento político en la educación contemporánea. Tras una anotación metodológica que clarifica mi posición para la organización de la literatura, me detendré en caracterizar diferentes posturas sobre cómo la educación matemática es política y, por lo tanto, se relaciona con el asunto de la democratización, la justicia social y la equidad. Para finalizar, recojo los puntos importantes de estas visiones y señalo algunas de las tensiones en este campo en el contexto de América Latina.

\section{Las matemáticas escolares y el progreso social}

Hoy en día sería difícil imaginarnos una escuela sin una cantidad considerable de matemáticas. No obstante, una revisión histórica revela que hace más o menos un siglo atrás no eran un área preponderante del currículo (Karp y Schubring, 2014). En primer lugar, a principios del siglo xx no todos los países del mundo tenían un sistema educativo masivo estructurado por el Estado y con cobertura de la población. La importancia de la educación masiva como estrategia de formación de la relación entre individuo-ciudadano y Estado es importante de tener en cuenta como una condición de posibilidad de la formulación de currículos nacionales como parte de la escolaridad obligatoria. Como afirman Meyer, Ramírez y Soysal (1992, p. 131), las características de la educación masiva como elemento de la cultura occidental moderna incluyen cinco elementos: (1) el énfasis en la socialización del individuo para hacerlo parte de la sociedad; (2) el deseo de expandir tal membresía a todos los individuos, (3) la articulación de una visión secular de progreso, (4) la formulación de currículos cada vez más estandarizados, y (5) la asociación entre la idoneidad en el currículo y el desarrollo personal y de la nación. Esto significa que los Estados-nación crecientemente se apropiaron de la educación como un elemento importante de gobierno (Tröhler, 2013). La educación se tornó un asunto político y de política pública para la formación de los ciudadanos deseados del Estado. 
En segundo lugar, la discusión sobre qué áreas del conocimiento deberían ser parte del currículo oficial se inclinaba más hacia considerar que las letras y las humanidades eran aquellas materias que mejor podrían forjar el espíritu del ciudadano. Schreiber (2014) por ejemplo analiza el desarrollo del currículo escolar en Luxemburgo como parte de la formación de la noción de Estado y de su ciudadano luxemburgués; ahí las materias técnicas como la aritmética ocupaban poco tiempo de estudio. Esto no significa que elementos de aritmética y de geometría a través del dibujo no fueran parte de planes de estudio, sino que solo un nivel muy básico y funcional era parte de la educación de las mayorías. Los temas de matemáticas más avanzados se reservaban para las escuelas militares o las nacientes escuelas de ingeniería o universidades. De hecho, en la transición al siglo xx las matemáticas y las nacientes ciencias naturales junto con sus aplicaciones se consideraban como conocimientos de menor categoría pues su carácter práctico y técnico no era de gran interés para la formación de un ciudadano virtuoso (ver por ejemplo, Giacardi y Scoth, 2014).

Fue también a finales del siglo XIX que se empezaron a encontrar discusiones sistemáticas en distintos países del mundo sobre la importancia de los conocimientos matemáticos y científicos como materias importantes para formar el intelecto del individuo y afrontar problemas reales. Si bien estos argumentos están presentes en los debates sobre el "ciudadano del siglo $x x^{\prime \prime} \mathrm{y}$ en la ineludible fuerza de estos nuevos conocimientos para las necesidades del siglo que estaba por comenzar (por ejemplo, Laisant y Fehr, 1899), fue durante el periodo posterior a la Segunda Guerra Mundial cuando se pudo trazar un cambio definitivo de visión de la educación y del papel de las matemáticas con respecto a la formación del ciudadano (Tröhler, 2015). Tras el desarrollo exitoso de muchas tecnologías de guerra gracias al trabajo colaborativo e interdisciplinario de grupos de científicos, el deseo de progreso propio del optimismo tecnológico hizo posible articular la idea no solo de que los nuevos conocimientos matemáticos-científicos-tecnológicos deberían ser parte central de la educación obligatoria masiva para todos, sino también de que tales conocimientos pueden ofrecer las capacidades mentales y de comportamiento para la fabricación del nuevo ciudadano (Valero, 2017).

Esta idea se entrelaza con la manera en que la educación masiva de la población se comienza a concebir -como sugieren Meyer et al. (1992) - como un asunto de manejo económico a través de su inclusión como variable importante en las nacientes teorías del capital humano. Para los países miembros de la Organización para la Cooperación y el Desarrollo Económicos (OCDE) y para otros que pertenecen a organizaciones de fomento al desarrollo social y económico, como el Fondo Monetario Internacional (FMI), la Organización de Estados Americanos (OEA) y la 
Organización de las Naciones Unidas para la Educación, la Ciencia y la Cultura (Unesco), el apoyo para la expansión educativa, la modernización curricular y el monitoreo del avance educativo medido a través de tecnologías evaluativas masivas fueron maneras concretas de asegurar que la inversión en educación resultara efectivamente en mejor capacitación y, por lo tanto, en aumento en el capital humano (García, 2003). El desarrollo de los nuevos currículos como aquellos propios de la época de las matemáticas modernas y sus distintas variaciones en países del mundo se asociaron con las políticas económicas de fomento a la educación (Tröhler, 2015). En ese contexto el apoyo de la OCDE y la Unesco al Seminario de Royaumont, evento clave en el desarrollo de las matemáticas modernas, es una evidencia de la inversión en el impulso a las nuevas tecnologías para generar una nueva educación matemática (Furinghetti y Giacardi, 2010). Además, a partir de la década de 1960 las mediciones de los estudiantes en matemáticas, y en lengua materna, comenzaron a tomarse como un indicador privilegiado para monitorear la efectividad de los currículos nacionales y de la instrucción en general (Postlethwaite, 1967; Rindermann y Ceci, 2009); y con esto su asociación con el progreso del individuo y de la nación, y al mismo tiempo su enmarañamiento en los sistemas de clasificación que seleccionan a aquellos exitosos y más aptos de aquellos que se convierten en un riesgo para sí mismos y para la sociedad (Lindblad, Petersson y Popkewitz, 2015).

No hay que olvidar en esta breve historización que la configuración de las ciencias educativas, en particular de la investigación en educación matemática, como un campo internacional preocupado por entender y mejorar los procesos de enseñanza y aprendizaje de las matemáticas, también ha sido una de las fuerzas destacadas en la articulación de la importancia de las matemáticas para la fabricación del sujeto escolar (Popkewitz, 2004). Como generadora del conocimiento científico para intervenir racionalmente en las prácticas pedagógicas con el diseño de mejores tecnologías para direccionar la cognición, la investigación forma parte de la red discursiva y de prácticas dentro de la cual la cualificación matemática ha adquirido un valor económico, social y cultural como indicador de progreso del individuo y de la nación (Llewellyn, 2015).

\section{Una anotación metodológica}

Esta breve mirada histórica permite evidenciar la configuración que hace de las matemáticas escolares un asunto cultural, político y económico. No porque la posesión de conocimiento o competencia matemática en los individuos sea una respuesta a la necesidad de los cambios sociales y económicos de una sociedad crecientemente industrializada y conectada, sino porque el creciente deseo de más y mejores cualificaciones matemáticas 
(y científicas) en la población es un asunto central en la consolidación de una idea de ciudadano que forma parte de las funciones productivas y políticas del Estado. Pero, ¡cuidado! Mi punto con esta observación no es confirmar que la educación matemática es o debería ser para generar democracia y más igualdad, equidad o justicia social. Aunque este fue uno de los puntos de partida de mi propia investigación (Valero, 1999) y ha sido un deseo de muchos profesores e investigadores que han adoptado las visiones críticas y políticas de la educación matemática, esta no es hoy en día mi punto de vista. Lo que quiero resaltar es el hecho de que la asociación entre las matemáticas escolares y la justicia social y/o equidad ha surgido en una configuración histórica contingente al proceso de expansión del poder del Estado-nación y la creación de nociones de ciudadanía. En palabras más sencillas: una cosa es decir que el poseer competencia matemática -para utilizar la terminología en boga- es un prerrequisito para la democracia, o que debería conducir a ella y que esto es un asunto de desarrollo y progreso, y otra cosa diferente es decir, que a lo largo de un siglo la idea de que el conocimiento de las letras y las humanidades era el fundamento de la virtud del ciudadano se ha reemplazado por la concepción del conocimiento matemático-científico-técnico como el centro de la virtud de un ciudadano que hoy en día es, además, fuerza laboral e incluso emprendedor. La primera idea es una afirmación de tipo normativo, típica de los análisis didácticos que formulan un sistema de razón para el "deber ser" de la práctica pedagógica. La segunda es una afirmación de tipo analítico, y apunta a cómo una noción que hoy en día parece tan incuestionable ha surgido como parte de la política cultural del currículo de matemáticas en sociedades contemporáneas.

Esta breve aclaración es importante pues indica que mi manera de proceder en esta revisión no obedece simplemente a la categorización de un barrido de la literatura, sino que se basa más bien en la idea de "investigar la investigación" (Pais y Valero, 2012; Valero, 2008). Esta es una posición que concibe la investigación no como una representación de la realidad social, sino como un elemento que forma y construye aquella realidad social al ser el conocimiento experto que formula maneras particulares de pensar y de actuar sobre los objetos y sujetos de investigación. En otras palabras, y como he argumentado en varios escritos (Halai, Muzaffar y Valero, 2016; Valero y García, 2014), la investigación en educación matemática genera marcos discursivos y racionalidades sobre lo que se considera como educación matemática en tiempos y lugares determinados. El surgimiento de la conexión entre educación matemática y justicia social y equidad es un ejemplo de esto, como se ha mostrado, y como se puede mirar en más detalle en discusiones sobre la emergencia de la idea de "matemáticas para todos" (Díaz, 2014; Valero, 2013). 
Por lo anterior, mi manera de organizar la creciente literatura de investigación que se ha producido internacionalmente frente a cómo y por qué la educación matemática se relaciona, o no, con la justicia y la equidad, se articula con la siguiente pregunta: ¿cuáles han sido las distintas posiciones teóricas y visiones sobre la relación entre educación matemática y democracia que se pueden identificar en la literatura, y cómo caracterizan esta relación? Dada la extensión de la literatura, en este momento es imposible dar cuenta exhaustiva de ella. Para más detalles, el lector puede dirigirse a recopilaciones sobre este tema (Atweh, Graven, Secada, y Valero, 2011; Forgasz y Rivera, 2012; Giménez, Díez-Palomar, y Civil, 2007; Jurdak, Vithal, Freitas, Gates y Kollosche, 2016; Keitel, 2013; Planas y Valero, 2016). A continuación, esbozaré dos posiciones distintas que es posible identificar frente a mi pregunta y haré explícita la racionalidad de estas posiciones.

\section{¡El empoderamiento para todos!}

Durante el siglo xx las matemáticas fueron sin lugar a duda un área de conocimiento central en la generación de ciencia y tecnología, y con ellas la posibilidad de los seres humanos de controlar y transformar su entorno natural, material y social. Los cambios en el mejoramiento de las condiciones de vida del ser humano han sido innegables y, por lo tanto, se hizo necesaria la inclusión de las matemáticas -no solo de la aritmética- como parte del currículo de la educación básica obligatoria. En sociedades cada vez más dependientes del desarrollo científico, la necesidad de que todos -o tantos como sea posible- aprendan matemáticas gracias a pedagogías apropiadas es un fin deseable del sistema educativo. Esto se condensa en la idea de las matemáticas empoderan a quien las aprende -ya sean niños, jóvenes o adultos-. La visión del empoderamiento tiene, sin embargo, diferentes matices, en especial en cómo el empoderamiento con y a través del aprendizaje de las matemáticas se relaciona con la justicia social y la equidad.

\section{El empoderamiento intrínseco}

Si el aprendizaje de las matemáticas empodera es porque hay algo esencial propio del conocimiento matemático en sí que se transfiere a quienes logran apropiarse de él. Tomado como una de las mejores expresiones del pensamiento humano, desde la antigua Grecia hasta nuestros días, la abstracción, la axiomatización, la formalización y el carácter verdadero de las matemáticas se han considerado como elementos de una forma de pensamiento que bien podía acercar al ser humano a los secretos del universo, o a la perfección de Dios, o a la forma más avanzada de cognición. La apropiación de estas características otorga a la persona una 
mejor capacidad de pensamiento y, por tanto, un empoderamiento. Y este se concibe en sociedades contemporáneas como un prerrequisito para la participación en la vida social, económica, cultural y política. Una discusión más detallada de este tipo de visión intrínseca del empoderamiento se encuentra en Skovsmose y Valero (2012).

Este tipo de visión por lo general se encuentra implícita en gran parte de las justificaciones para la mejora de la enseñanza de las matemáticas que están presentes tanto en documentos de política educativa nacional como en programas de formación y en la misma investigación educativa. Un ejemplo de este tipo de visión se refleja claramente en las iniciativas recientes de reforma en los Estados Unidos bajo la bandera de los Common Core Standards [Estándares comunes centrales], una iniciativa promovida por grupos de matemáticos universitarios para dar respuesta a los problemas del bajo rendimiento en matemáticas en ese país. Como resultado de lo que se ha llamado la "guerra de las matemáticas" (Schoenfeld, 2004), que fue una reacción a las iniciativas del Consejo Nacional de Profesores de Matemáticas (NCTM) en los años noventa, se ha promulgado la necesidad de volver a las ideas centrales de las matemáticas como el eje articulador de nuevos currículos que en verdad puedan balancear calidad con equidad. El argumento es que la variedad de contenidos y la calidad con que se enseña en diversas escuelas en los Estados Unidos son un factor muy fuerte de disminución de las oportunidades de aprendizaje de los estudiantes. La oportunidad de aprendizaje se define como la posibilidad de acceder a contenidos apropiados, pues a la larga es el aprendizaje de contenidos específicos lo que se considera el objetivo central de la educación matemática. De ahí que los Common Core Standards sean percibidos como una iniciativa que definitivamente puede abordar el problema de equidad en términos de la persistente brecha en la diferencia de resultados escolares de distintos grupos de la población. Además, es una solución al bajo puntaje de los estudiantes estadounidenses en estudios internacionales comparativos como el tIMms (Schmidt, Cogan y McKnight, 2010).

Este es un ejemplo de un tipo de argumento que, al enfocarse en el contenido matemático mismo y en su aprendizaje, sitúa el asunto del empoderamiento con relación a las características mismas del conocimiento matemático y al hecho de que este puede potenciar al individuo y sus capacidades y oportunidades educativas. Basta decir que esta idea no solo ha estado presente en Estados Unidos sino que, dada la legitimidad de quienes la han abanderado, se ha expandido a muchos otros países latinoamericanos en situaciones donde se enfatiza la idoneidad matemática del profesor y la necesidad de volver a los contenidos duros como la esencia de la recuperación de la educación matemática. 


\section{El empoderamiento en los usos y aplicaciones}

El empoderamiento no se fundamenta en lo que el conocimiento matemático otorga al individuo, sino en la capacidad del individuo para usar el conocimiento en el abordaje de problemas. La visión de la utilidad del conocimiento en el abordaje de problemas y de la educación como una actividad pragmática de relacionamiento con el mundo social se traza en la transición entre el siglo xIx y el xx. Una serie de pensadores de ese tiempo enuncian esta idea; entre ellos, la filosofía pragmática del John Dewey (1916) se ha reconocido como un punto importante en la orientación del conocimiento escolar y la educación como elementos de actividad y transformación del individuo y la sociedad.

En educación matemática gran parte de las tendencias que abogan por la visión de las matemáticas como una actividad de resolución de problemas y las pedagogías de indagación, que comparten la idea del conocimiento-en-uso, se alinean con esta visión. En su conceptualización sobre la educación matemática basada en la indagación (EBI), Artigue y Blomhøj (2013) señalan que

... uno de los objetivos esenciales del EBI ha sido promover los valores de emancipación y democracia. Este objetivo afecta los asuntos que se consideran significativos, especialmente por la importancia que se les da a las conexiones con las preocupaciones de la vida real, al igual que la manera como estos asuntos se abordan en el proceso de indagación, en particular la visión de las relaciones ente diferentes actores dentro y fuera de la escuela que pueden potencialmente involucrarse en el proceso. (p. 808, traducción propia).

Igualmente, gran parte de la tendencia de la modelación en educación matemática pone de relieve una visión de las matemáticas como conocimientos y procedimientos en acción, y uso en la construcción de modelos matemáticos como parte de sistemas científicos, tecnológicos y sociales de acción, a través de los cuales se abordan problemas complejos (Stillman, Blum, y Biembengut, 2015). El propósito principal es facilitar que se comprendan las matemáticas como una herramienta poderosa que se puede conectar con una diversidad de aspectos de la vida natural y social. Así la adquisición de la competencia de modelación matemática "potencia el conocimiento, pero también asegura el avance de la sostenibilidad del bienestar de la salud, la educación y el medioambiente, y la reducción de la pobreza y la desventaja" (Niss, Blum y Galbraith, 2007, pp. 17-18, traducción propia).

Recientemente la visión de la emancipación a través de la utilidad de las matemáticas en la modelización se ha criticado por promover un mito de equidad y acceso a oportunidades de aprendizaje de las matemáticas. 
Jablonka y Gellert (2011) analizan los supuestos sobre los cuales una serie de iniciativas de modelización basan su visión de competencia y pedagogía. Ellos concluyen que el énfasis que se presta a la utilidad y aplicación del conocimiento matemático sigue asociándose a una visión a-cultural de la actividad matemática. Esto aparentemente es contradictorio: que las herramientas matemáticas se apliquen en el contexto de un problema, no quiere decir que en el fondo de las propuestas de modelación exista una posición ontológica diferente a las posiciones platonistas que ubican los objetos y herramientas matemáticas fuera del campo de las relaciones culturales e históricas de la humanidad. Esto, plantean Jablonka y Gellert, deja el espacio abierto para promover agendas no necesariamente alineadas con la equidad y democratización, como suponen Artigue y Blomhøj (2013) y Niss et al. (2007) en las afirmaciones mencionadas. Además, cuando la actividad misma de la modelización matemática no se concibe como una actividad cultural en sí, se mantiene una idea de la neutralidad ética de las prácticas de modelización matemática. De esta manera, el mito creado fácilmente deja abierta la puerta para que la educación matemática prepare jóvenes que serán los futuros trabajadores eficientes de un orden neoliberal, donde las matemáticas y las ciencias tienen un valor de mercado que sobrepasa la posibilidad de que los jóvenes se involucren de verdad en la transformación de un mundo de desigualdad. De esta manera, la promoción de un lenguaje de "democracia" fácilmente puede resultar en la generación de más inequidad.

\section{El empoderamiento crítico}

El empoderamiento no está en las características intrínsecas de las matemáticas ni en la capacidad de darles uso, ni tampoco en que en las sociedades modernas las dos anteriores se consideren deseables. El foco de lo político está en la posibilidad de reconocer no solo los efectos positivos de las matemáticas en la construcción de bienestar y progreso, sino también en la generación de destrucción y riesgos para el ser humano y la sociedad. El empoderamiento es crítico si duda de la neutralidad de las matemáticas y ellas se comienzan a ver en su relación con críticas al optimismo tecnológico y la fe en el progreso como narrativas importantes de las ideas de desarrollo moderno.

El trabajo de Skovsmose (1999) es ejemplar dentro de esta perspectiva. La idea de la educación matemática crítica como un enfoque filosófico para la educación matemática plantea que una de las competencias más importantes que debería resultar de la educación matemática es la competencia democrática asociada con la alfabetización matemática. Esto se refiere a la posibilidad de tomar una distancia crítica de la manera como las matemáticas, a través de su participación en la formulación de los

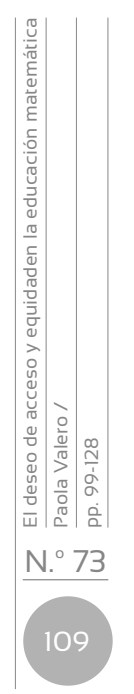


múltiples modelos sobre los que se basa la sociedad en sus distintos aspectos, genera tanto maravillas como horrores. Más aún, para un ciudadano y su participación en procesos democráticos, tal distancia está en poder ver más allá de los hechos para pensar en las decisiones que se toman diariamente con base en tales modelos expertos matemáticos.

En una línea similar Frankenstein (1995) y Gutstein (2006) han señalado no solo los fundamentos teóricos sino también los principios de pedagogías donde se evidencia la crítica a las estructuras sociales con y a través de las matemáticas. Para ellos el problema de la diferenciación de clase y las estructuras de segregación racial es importante de abordar en el aula misma de matemáticas. El empoderamiento crítico está en el estudio, a través de esta disciplina, de las situaciones sociales que los estudiantes de bajo nivel socioeconómico y pertenecientes a grupos raciales segregados viven a diario. Este tipo de ideas también han tomado fuerza en la corriente de la modelización sociocrítica que, por medio del trabajo de distintos grupos de investigación en Brasil (Araujo, 2009; Barbosa, 2006), han expandido las ideas y posibilidades de práctica educativa, al enfatizar el conocimiento reflexivo sobre el funcionamiento de las matemáticas en las prácticas sociales y la posibilidad de una concientización política de los estudiantes. En Colombia, Camelo et al. (2013) examinan las posibilidades y tensiones de la introducción de escenarios de modelización donde los jóvenes de comunidades urbanas "en riesgo" se enfrentan a la modelización, el análisis y la propuesta de soluciones a situaciones que atañen a su bienestar y el de su comunidad (como lo son el manejo de residuos orgánicos en la zona donde viven en la cercanía de una gran mercado de distribución de alimentos en Bogotá). A partir de su trabajo concluyen que el entrelazamiento entre las actividades matemáticas de modelización y otros elementos disciplinares, sobre todo su concientización política, tienen un potencial crítico de empoderamiento.

\section{Desigualdades y educación matemática}

En el periodo de optimismo tecnológico de la posguerra se conformaron grupos interdisciplinarios de expertos educadores, psicólogos y evaluadores que construyeron mediciones del rendimiento escolar (por ejemplo, International Association for the Evaluation of Educational Achievement, 2016). Estas tecnologías de medición fueron centrales en la configuración de la idea de que el rendimiento escolar en matemáticas podía utilizarse como una medida de desarrollo individual, grupal y nacional Jablonka, 2016; Valero y Meaney, 2014). De ahí que el deseo de las "matemáticas para todos" no solo se convirtió en un elemento explícito de las políticas educativas para justificar la necesidad de mejorar la enseñanza de las matemáticas escolares, sino que también contribuyó a la construcción del fracaso en las matemáticas escolares como 
un hecho que hay atacar, y un problema de investigación que hay que abordar. A partir de la década de 1980 el rendimiento diferencial de distintos grupos poblacionales se empezó a considerar como un "problema" de la educación matemática y de su investigación. Numerosos recuentos del surgimiento de las visiones sociales (Lerman, 2000) y políticas de la educación matemática (Gutiérrez, 2013; Valero, 2012b) apuntan al V Congreso Internacional de Educación Matemática (ICME 5) en Australia como uno de los primeros momentos oficiales de la comunidad internacional donde se trató el problema de que las formas de enseñanza y los currículos existentes hasta el momento se fundamentaban en una visión elitista de las matemáticas que generaban el cierre sistemático de acceso a muchos aprendices en el mundo. El reporte Mathematics for all (Damerow, Dunkley, Nebres y Werry, 1984), financiado por la Unesco, fue la manifestación del surgimiento de la idea de que las matemáticas, aquello tan deseado como motor del progreso social, actúan como "filtro al acceso educativo" de un sinnúmero de estudiantes. Así, el asunto del fracaso escolar sistemático en matemáticas pasó de ser una preocupación económica, a ser una preocupación política y sobre todo democrática.

La creciente adopción de marcos conceptuales de otras ciencias sociales diferentes a la psicología cognitiva para pensar los procesos de enseñanza y aprendizaje de las matemáticas resultó en una diversidad de posibilidades de análisis e intervención en el problema del acceso y éxito diferencial en esta área. La manera como distintos aspectos de la educación matemática crean ventajas y desventajas para distintos grupos sociales se convirtió en un asunto de acceso equitativo a la educación y a los beneficios sociales, económicos y culturales asociados (Jablonka, 2009). La discusión sobre quiénes son aquellos que fracasan sistemáticamente se ha dejado de entender netamente como un asunto de deficiencia cognitiva individual (Frade, Acioly-Régnier, y Jun, 2013). Gran parte de la investigación sobre desarrollo cognitivo en matemáticas hasta ese momento se basaba principalmente en la visión ontogenética piagetiana. Las grandes y modernas tecnologías de la educación matemática que acompañaron y siguieron la formulación y expansión de currículos modernos -incluido el periodo de la matemática moderna y algunos desarrollos posteriores como el currículo de la renovación curricular en Colombia (Vasco, 2011)- adoptaron la idea de que existe una evolución natural y normal del pensamiento del niño. Este niño es un sujeto cognitivo, es decir, "un sujeto intrínsecamente racional, autosostenido que madura a medida que interpreta y refina la retroalimentación que se supone ser éticamente neutral del medioambiente" (Radford, 2008b, p. 216, traducción propia). Este ser es un representante universal de la razón humana, y su pensamiento se desarrolla a través de un aprendizaje adaptativo, que tiende al pensamiento adulto, en especial aquel ejemplificado por el pensamiento matemático adulto maduro. De ahí que la labor de las matemáticas escolares

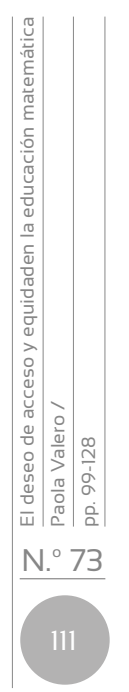


sea moldear el desarrollo del individuo para encaminarlo, tan cerca como sea posible, al modelo general de racionalidad. Dada esta lógica, las fallas en el logro de los parámetros esperados estipulados en planes curriculares obedecen o bien a fallas en la instrucción (que la investigación debe intentar corregir) o a fallas en el individuo mismo. La generación de todo un discurso de la deficiencia forma parte de este tipo de lógica, y ha sido una de las batallas de investigadores que, adoptando otras teorías sociales, culturales y políticas, han buscado otro tipo de explicaciones al fracaso escolar en matemáticas.

Desde los años noventa hasta el momento se podría decir que la investigación y práctica educativa que busca explicaciones y acciones alternativas al discurso de la deficiencia ha crecido notablemente. Toda esta corriente se preocupa por explorar cómo el acceso y éxito diferencial en matemáticas no son un asunto de deficiencia cognitiva individual, sino más bien de cómo las visiones del contenido, las prácticas pedagógicas y las configuraciones institucionales que conforman la red de prácticas de la educación matemática (Morgan, 2014; Valero, 2012a) generan un acceso diferenciado a distintos tipos de estudiantes en relación con categorías de diferenciación como son la habilidad, el género, la raza, la etnicidad, el lenguaje, el nivel socioeconómico, la clase social e incluso la afiliación confesional. A continuación, delinearé dos posiciones presentes en la literatura sobre cuáles son las fuentes de la desigualdad y dónde se pone en operación.

\section{La desigualdad epistemológica}

A través de la historia, la cultura occidental eurocéntrica ha construido una narrativa de superioridad de sus formas de conocimiento frente a las formas de conocimiento de muchas otras culturas (Harding, 1998; Radford, 2008a). En la configuración de la episteme clásica de la modernidad de los siglos XVII y XVIII, la mátesis o "la ciencia universal de la medida y el orden" (Foucault, 1973, p. 56) es un elemento central que establece relaciones de orden entre las cosas, incluso aquellas que no son medibles. Medida y orden en la episteme clásica moderna no se separan de la clasificación. Y ninguna de ellas surge en un vacío, sino en una red de prácticas materiales y discursivas que ubican al conocimiento como un elemento importante del gobierno y, por lo tanto, de poder. El siglo xvı fue también el gran siglo de la empresa colonial europea, y las clasificaciones y ordenaciones con y a través del conocimiento estuvieron en el centro de la lógica comparativa que posiciona el conocimiento del colonizador por encima del de los colonizados. La Colonia con su red de explotación económica instauró también la idea de la superioridad de la cultura europea, de sus formas de vida y de sus conocimientos. En esta red fueron co-emergiendo y entrelazando la formación de las nuevas ideas matemáticas con la narrativa de las Matemáticas 
-con M mayúscula, siguiendo la sugerencia de Bishop (2005)- como ese campo del saber universal y cierto. Esta narrativa también implica que otras matemáticas -con $m$ minúscula- están subordinadas a la Matemática.

Sustentar todos los detalles de esta idea sobrepasa los límites de esta revisión. No obstante, el punto central es que la configuración histórica de una narrativa de superioridad epistemológica de las matemáticas dentro de un orden cultural de ordenación de distintos pueblos y sus culturas se puede identificar justamente como la fuente de la desventaja epistemológica. En términos educativos esto quiere decir que aquellas formas de pensamiento o conocimiento propios de otras culturas y formas de vida que se distancien de las de la cultura blanca europea, dentro del orden cultural y político establecido, se consideran como epistemológicamente inferiores. Así, el fracaso escolar de grupos particulares de estudiantes en su apropiación de las Matemáticas que forman parte de los currículos oficiales de la educación masiva se explica por su posición de inferioridad epistemológica frente al patrón de conocimiento que impone la noción de lo que es normal y deseado. Knijnik (2012) ha presentado una crítica a este tipo de diferenciación política de diversas formas de vida y sus matemáticas.

La investigación en etnomatemática ha sido importante para entender el supuesto de la desigualdad epistemológica. Parte de su proyecto político como estudio de las técnicas y prácticas de conocimiento de distintos grupos culturales (D'Ambrosio, 2006, 2011) ha sido presentar una posición crítica frente a la hegemonía y supremacía de las Matemáticas occidentales para abrir campo a muchas otras formas de conocimiento enraizadas en diferentes mundos culturales (Gerdes, 2007). Parte del gran programa de la etnomatemática -que se ha expandido en las últimas décadas en muchos países latinoamericanos- ha sido estudiar las diferencias y similitudes entre los conocimientos oficiales de la escuela y los conocimientos en las prácticas culturales, para proponer pedagogías que no posicionen en desventaja a niños y adultos pertenecientes a tales grupos frente a la posibilidad de apropiarse de sus conocimientos matemáticos y los de la cultura dominante. Ejemplos de este trabajo con diversas comunidades indígenas, campesinas y de práctica (como carpinteros o vendedores de mercado) hay muchos en América Latina, y se pueden encontrar entre otras en la Revista Latinoamericana de Etnomatemática.

Trabajos más recientes tratan de mover el entendimiento del supuesto de desigualdad epistemológica de las matemáticas de diversos grupos y, adoptando una posición teórica decolonial, abordan el problema preguntándose por la posibilidad de una hibridación de conocimientos y simetría en las relaciones entre las Matemáticas occidentales y las matemáticas de otros grupos culturales en su encuentro. Parra (2017) afirma que la persistencia del supuesto de inferioridad epistemológica corre el riesgo de seguir vivo en visiones de la etnomatemática que conciben tal programa

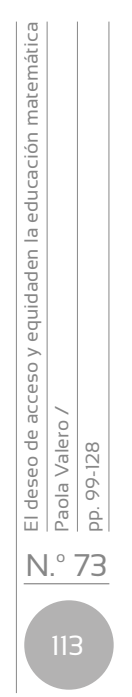


como una intersección entre matemáticas y cultura. Con la metáfora del "trueque", se reta a ir más allá en el encuentro de (M)matemáticas para construir posibilidades de intercambio donde el conocimiento matemático pueda interrogar y nutrir al de las Matemáticas. El objetivo de tal trueque es mostrar que las Matemáticas no tienen un lugar privilegiado, sino que pueden "referirse a algunos de sus objetos en formas no familiares para ganar nuevos entendimientos" (p. 102, traducción propia). El objetivo es desmitificar las Matemáticas y mostrarlas como algo "mundano, como cualquier otro asunto" (p. 102, traducción propia).

Mientras que muchos estudios han mostrado que las ciencias naturales son construcciones culturales no solo eurocéntricas sino también masculinas (Chassot, 2003; Harding, 1998), no muchos estudios similares existen con respecto a las matemáticas. Una compilación de algunos de ellos se encuentra en Forgazs y Rivera (2012). No obstante, se ha apuntado a que las diferencias en el rendimiento de mujeres y hombres -generalmente a favor de estos- y la mayor participación de los hombres en la producción misma de las matemáticas y en profesiones de ciencia y tecnología no se deben a ninguna diferencia ni física ni intelectual de las mujeres, sino más bien a barreras culturales (Fennema, 1995). Con base en epistemologías feministas se propone examinar la constitución histórica de las matemáticas como un dominio masculino (Damarin, 1995), producido no solo en su mayoría por hombres sino también en configuraciones de sociedades patriarcales donde lo femenino se posicionó como el "otro" inferior en fuerza, cuerpo e intelecto. Chronaki (2008) evidencia cómo las mismas prácticas científicas emergentes en ciencias biológicas, genética y antropometría de finales del siglo xix generaron categorías de género y raza donde a los seres pertenecientes a las categorías "mujer" o "no-blancos" se les asignaron las "características primitivas de cráneos estrechos, cuasi-infantiles y delicados que se encuentran en las castas inferiores, diferentes a las cabezas más robustas y redondeadas de los hombres de razas superiores" (p. 17). Este tipo de concepción está en la base del supuesto de que seres con tales características no son aptos para apropiarse del conocimiento matemático más avanzado que, por tanto, solo puede ser producido por las mentes masculinas "superiores" y solo puede ser totalmente apropiado y recreado por ellas. Chronaki (2011) muestra cómo estas ideas están presentes en las afirmaciones de jóvenes (hombres y mujeres) y sus profesores sobre su aptitud y habilidad para ser parte de ambientes matemáticos y tecno-científicos.

Este tipo de nociones no son "verdades" eternas e inamovibles. Mi punto al traer esta discusión de la desigualdad epistemológica es señalar que la explicación del fracaso en matemáticas de distintos grupos de la población como un asunto de deficiencia cognitiva se relaciona con la permanencia del supuesto de que existe una supremacía del conocimiento 
matemático frente a otras formas de conocimiento y frente a otros que no lo han generado. La investigación que aborda este tipo de supuesto histórico ofrece otras posibilidades para concebir la labor de educar matemáticamente manteniendo un ojo crítico sobre cómo la verdad a menudo no cuestionada de la supremacía de las matemáticas es la fuente de diferenciación de individuos.

\section{La reproducción de las desigualdades sociales}

La fuente de la desigualdad en los logros en matemáticas está en las desigualdades sociales mismas, y en la función reproductora de la escuela y las pedagogías convencionales que se adhieren implícitamente a la visión de la supremacía del conocimiento matemático (y de sus creadores). En las sociedades contemporáneas existen una serie de mecanismos de orden y clasificación de la población, sobre la cual se basa la distribución desigual de acceso a recursos, beneficios y posibilidades de vida. Como parte de los procesos históricos de gobierno y manejo de las poblaciones, se han creado diferenciaciones en cuanto a habilidad, género, raza, lenguaje, clase social, estatus socioeconómico, etnicidad, religión, orientación sexual, etc. Estas diferencias no son generadas exclusivamente en la educación, pero esta además de reproducirlas, las refuerza. Más aún, las pedagogías y los procesos educativos que son parte del currículo de matemáticas tienden con frecuencia a contribuir a tal reproducción y a exacerbar las desigualdades entre estudiantes y sus posibilidades, dado el valor social atribuido al buen rendimiento en matemáticas.

Como parte del viraje hacia lo social y lo político antes mencionado, la investigación en educación matemática que se ha enfocado en analizar, entre otros, el fracaso escolar, el papel de filtro de las matemáticas escolares y el fenómeno de las "brechas de rendimiento" entre distintos grupos de la población, ha adoptado una variedad de marcos teóricos de disciplinas como la sociología, la psicología social, la antropología, los estudios culturales y multiculturales. Estos marcos se han recontextualizado al estudio de la educación matemática (Jablonka, Wagner y Walshaw, 2013; Lerman, 2006). Es importante decir que gran parte de los estudios de este tipo se han producido en países de habla inglesa, y que muchos de ellos no solo tratan de proveer un análisis de los problemas mismos, sino en especial de proponer pedagogías alternativas que promuevan la inclusión. De ahí que en estos estudios las conceptualizaciones de ideas como democracia, equidad, igualdad, justicia social, inclusión o exclusión varían. Lo que es común es el deseo por presentar una visión de cuestionamiento frente a la idea de que el fracaso escolar en matemáticas es "natural", y que las prácticas pedagógicas son neutrales. A continuación, me enfocaré en 
algunas de las áreas de investigación y sus ideas principales con respecto a cómo las desigualdades sociales se reproducen en los procesos de la educación matemática.

En la sección anterior se hizo referencia al supuesto de la diferencia epistemológica de distintos grupos culturales frente a las matemáticas. Las diferenciaciones de raza y etnicidad-que en sí mismas son objeto de estudios culturales (por ejemplo, Wade, 2010)- se han estudiado con respecto a la educación matemática. La noción de raza permanece en los discursos populares como una diferenciación biológica objetiva de fenotipos humanos y no como una noción cuyo significado ha variado en el tiempo y que hoy en día se entiende como una categoría inseparable de la manera como el hombre blanco europeo, a través de la historia, ha pensado en su diferencia sobre los otros tipos de personas con quienes se ha relacionado. La noción de etnicidad, más recientemente usada en discusiones académicas de diferencia a partir de la posguerra, desde cuando la noción de raza perdió su aparente objetividad biológica, se refiere con frecuencia a grupos culturales minoritarios con respecto al grupo mayoritario que constituye la base de un Estado-nación (Wade, 2010, pp. 12-15). Muchos de estos estudios inician con la constatación de que grupos raciales y étnicos determinados muestran un rendimiento inferior a lo esperado. A través del estudio de las prácticas de enseñanza y aprendizaje de las matemáticas en el aula y de cómo se generan posiciones y narrativas de identidad de los estudiantes como menos hábiles, desinteresados e incompetentes, muchas de estas investigaciones documentan cómo los mecanismos de segregación racial y étnica operan en las configuraciones institucionales de las matemáticas escolares.

Doğan y Haser (2014), por ejemplo, muestran cómo, en el currículo oficial de matemáticas de Turquía y en los libros de texto con sus objetivos, tipos de ejercicios y recomendaciones para la práctica de los profesores, se promueve una visión de una vida de clase media, empresarial, nacionalista turca como el ideal que todo estudiante debe alcanzar. Esta visión dificulta la identificación de estudiantes de otros grupos sociales, en especial de minorías kurdas, con las normas de participación y éxito en las matemáticas escolares. Casos similares se han documentado para la población inmigrante en Estados Unidos, en particular los latinos (Civil, 2007); en muchos de los países europeos frente a los inmigrantes recientes (Alrø, Skovsmose y Valero, 2008; Planas y López, 2007); y frente a las poblaciones indígenas minoritarias en Australia y el Pacífico (Meaney, Edmonds-Wathen, McMurchy-Pilkington y Trinick, 2016) y en América Latina (Secada, Cueto y Andrade, 2003).

La investigación sobre la segregación de estudiantes afroamericanos en Estados Unidos es de particular interés pues ofrece herramientas teóricas para hacerle frente a la manera como los mecanismos de diferenciación 
racial forman parte de los espacios institucionales. Martin (2011) usa la noción de espacio institucional blanco para referirse a la manera como la serie de espacios y prácticas de la educación matemática en Estados Unidos se basan en el supuesto de la inferioridad epistemológica de los grupos afroamericanos. Su investigación ha sido de inspiración para examinar otros contextos, como los de la educación de las minorías afrodescendientes en Colombia. Valoyez y Martin (2016) señalan que la ideología de la mezcla racial que domina en países latinoamericanos como Colombia se asocia con la idea de que todos, independientemente de su raza y color, tienen iguales oportunidades. Esta idea enmascara, no obstante, el hecho de que en la nación "mestiza" donde se promueve una identidad racial unificada, las instituciones sociales continúan reproduciendo una valorización diferenciada y racializada de distintos grupos sociales. Esto se evidencia claramente en, por ejemplo, las narrativas de profesores sobre los niños afrodescendientes como "anormales" y no aptos para el éxito escolar en matemáticas, pues sus "comunidades están poco interesadas en desarrollar [el pensamiento matemático]" (Valoyez-Chavez, 2014, traducción propia).

Otra serie de estudios se enfocan en entender la relación entre clase social o posición socioeconómica y rendimiento diferenciado en matemáticas. Hoy en día es un hecho que cuanto más abajo en el ordenamiento socioeconómico se encuentren un estudiante, su familia y comunidad, menor se espera que sea su rendimiento escolar. En muchos países del mundo desde los años sesenta ha existido una serie de modelos sociológicos y matemáticos que relacionan el resultado educativo, medido a través de pruebas estandarizadas en matemáticas y lenguaje, con el nivel socioeconómico, medido a través de una serie de variables asociadas, como el ingreso de los padres, los recursos culturales como libros en casa, etc. (Baker, Goesling y LeTendre, 2002; Coleman et al., 1966; Heyneman y Loxley, 1982). Recientemente esta relación fue el tema de un grupo internacional de estudio en educación matemática (Valero et al., 2015; Valero y Meaney, 2014) donde se trató de problematizar estos estudios estadísticos que, aunque informativos, no hacen visibles los mecanismos de las prácticas sociales que generan desigualdad educativa en matemáticas. Estos estudios y muchos otros anteriores se concentran en estudiar cómo las prácticas pedagógicas mismas de las matemáticas generan los resultados diferenciales.

Una posición ya clásica ha adoptado la sociología educativa de Pierre Bourdieu (Bourdieu y Passeron, 1990) y de Basil Berstein (1993) para evidenciar que, como parte de la escuela, las prácticas pedagógicas de las matemáticas escolares adoptan las normas culturales de la clase media y que, por tanto, los estudiantes de clases bajas no cuentan con el capital social y cultural para poder decodificar el juego que le propone el profesor 
en la escuela. Y esto es porque en una situación escolar es difícil separar lo que es puramente matemático del resto de normas culturales y hábitos lingüísticos que permean las actividades del aula. Por eso, el fracaso no es necesariamente un asunto de discapacidad cognitiva matemática, sino más bien de dificultad en "romper el código" de conducta que la escuela impone para considerar a un estudiante "exitoso" (Jorgensen, Gates y Roper, 2014). Más aún, a medida que los estudiantes se mueven a niveles más avanzados de matemáticas, el lenguaje vertical especializado que caracteriza el registro matemático conceptual entra en conflicto con las formas discursivas más horizontales a las cuales los estudiantes de clase baja están acostumbrados como parte de su participación en la vida fuera de la escuela. Este conflicto es el que muchas veces les imposibilita poder participar como se espera en prácticas matemáticas más avanzadas. La conclusión de muchos de estos estudios es, en general, que la estructuración de las prácticas pedagógicas como parte de sociedades diferenciadas por clase social y nivel socioeconómico ordena y clasifica a los estudiantes, manteniéndolos en su posición de desventaja.

Además de los marcos teóricos de la sociología, el concepto de identidad junto con visiones socioculturales del aprendizaje han sido importantes para entender la diferenciación en el aula de matemáticas. Con base en teorías discursivas, Sfard y Prusak (2006) propusieron la idea de que la identidad, o el sentido que todos tenemos de quiénes somos, no depende de una esencia del ser individual, sino que más bien es un constructo cambiante en la relación entre la persona y los otros y el mundo social y cultural. En una enunciación provocadora plantearon que la identidad no es otra cosa que los enunciados que cada uno de nosotros dice sobre sí mismo y que los demás también emiten sobre uno. La noción de identidad se convierte en el "eslabón perdido" que conecta al individuo con lo social y cultural, y es aquel vínculo que se había tratado de tejer entre las teorías que separan la cognición individual y aquellas que apuntan a lo social y cultural como fuentes de conocimiento. La noción de identidad ha sido una herramienta teórica muy importante para investigar cómo las prácticas escolares de matemáticas sistemáticamente posicionan a unos estudiantes como "buenos" y a otros como "malos" para matemáticas y así afectan el sentido de ser de los estudiantes (Stentoft y Valero, 2009). Ellos mismos y el entorno, ya desde cuarto grado de primaria, producen narrativas de identidad de sí mismos como incapaces, deficientes, aburridos y con odio hacia las matemáticas, y por esto se ven a sí mismos casi como personas sin futuro (Darragh, 2016; Lange, 2016). Este tipo de narrativas son muy serias y difíciles de romper una vez se instalan en el imaginario de los estudiantes, sus profesores, sus compañeros, e incluso padres de familia. Cobb y Hodge (2007) anotan cómo estas narrativas de identidad actúan como profecías sobre el futuro de cada estudiante que, de tanto repetirse a lo largo de los años de escuela, terminan volviéndose verdad. 
En esta revisión sería imposible cobijar todos los estudios que apuntan a las diferenciaciones por lenguaje, que en el último tiempo han crecido particularmente en países europeos dada la inmigración de poblaciones que no manejan la lengua de instrucción (Norén, 2015). El Estudio 21 del ICMI sobre multilingüismo y educación matemática presenta un buen barrido de trabajos existentes en situaciones donde la presencia de múltiples lenguajes en el aula de matemáticas se convierte potencialmente en un factor de diferenciación y desigualdad (Barwell et al., 2016). Una vez más, en estos estudios no solo hay un intento por ofrecer explicaciones de corte social y cultural al fracaso escolar sistemático de ciertos grupos de estudiantes, sino que también se ofrece el análisis de cómo distintas alternativas pedagógicas y organizaciones institucionales del currículo pueden mitigar los efectos negativos de los mecanismos sociales de diferenciación operantes en la escuela.

\section{Un campo de tensiones}

En la revisión anterior se estableció como punto de partida que el creciente deseo por más cualificaciones matemáticas en los ciudadanos es parte de una configuración histórica, cultural y política reciente. Tal deseo conecta a la educación matemática con nociones como democracia, in(ex)clusión, desigualdad, equidad y justicia social. Si bien identifiqué las distintas posiciones que han aparecido en la literatura sobre cuál es esta relación, no presenté un sentido preciso a estas últimas nociones. Lo que pueden significar estas ideas en educación matemática ha sido discutido anteriormente por Skovsmose y Valero (2012) o más recientemente por Aguilar y Zavaleta (2012) para el caso de la democracia, o por Healy y Powell (2013) o por Secada (2011) para el caso de equidad y desigualdad, o por García et al. (2013) para el caso de la in(ex)clusión. Estos conceptos varían de acuerdo con las diferentes posiciones teóricas adoptadas por los investigadores, y de acuerdo con tiempos y espacios donde se estudia la relación. Es decir, estas nociones, tanto como lo que es la educación matemática misma, se escapan de la ilusión de una definición precisa y universal de su significado.

A pesar de esto, lo que parece ser persistente es, por un lado, el creciente deseo de más conocimientos, herramientas y competencias matemáticas, y, por el otro, el consistente logro diferenciado en las matemáticas escolares. De hecho, muy pocos alcanzan los niveles superiores y una proporción casi constante entre 16\% y $20 \%$ de la población logra alcanzar los niveles mínimos deseados, de acuerdo con las mediciones nacionales o internacionales. Esta tensión entre el deseo y el logro ha pasado a ser un asunto de política educativa en la medida en que, desde mitades del siglo $x x$, el logro individual, grupal, nacional e internacional se ha convertido

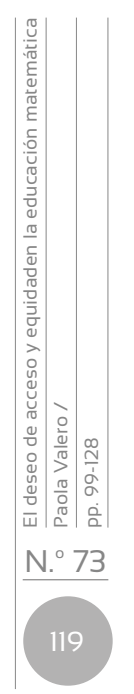


en un indicador de capital humano, y con esto, de desarrollo y bienestar social y económico. El resultado es entonces la configuración del bajo rendimiento en matemáticas como un asunto de equidad y de democracia. La gran tensión se encuentra en que el deseo por más matemáticas como un asunto de bienestar social y de democracia genera una clasificación, un ordenamiento y una exclusión cada vez más brutal de las personas de acuerdo con sus capacidades matemáticas.

En la revisión hice énfasis en las conceptualizaciones y racionalidades que se pueden identificar en las diversas posiciones sobre este tema. Privilegié una postura más analítica y dejé en un segundo plano el énfasis en la descripción de iniciativas y recomendaciones para remediar o compensar las desigualdades en la práctica de la enseñanza y aprendizaje de las matemáticas. En las recopilaciones que he mencionado se encuentran las descripciones y análisis de distintas innovaciones pedagógicas e iniciativas curriculares que tienen por objetivo mejorar la participación y los resultados de estudiantes en posiciones desaventajadas. Muchas de estas iniciativas han documentado efectos positivos. El no haberlas señalado no quiere decir que no sean importantes.

No obstante, mi intención al no detenerme en ellas es cuestionar la narrativa de salvación que se asocia a las matemáticas como aquello que mejora al ser humano y a la sociedad en general, dada su superioridad epistemológica y su poder como herramienta para competir en una economía del conocimiento globalizada. Esta narrativa se ilustra en análisis como los de los resultados de las pruebas PISA, cuya maquinaria cada vez más influye en las políticas educativas nacionales. Según este análisis, los ciudadanos

... con habilidades sólidas en matemáticas se inclinan más a ofrecerse como voluntarios, a verse a sí mismos más como actores que como objetos de procesos políticos, e incluso son más dados a confiar en otros. Justicia, integridad e inclusión en la política pública dependen completamente de las habilidades de los ciudadanos. (OECD, 2014, p. 6, traducción propia).

¡Esto quiere decir que las habilidades matemáticas incluso otorgan características morales y éticas a las personas, a tal punto que esas características se transferirán a las políticas públicas en forma de justicia, integridad e inclusión! La adopción implícita de estas narrativas nos sitúa fácilmente un espacio donde la lógica de la exclusión con las matemáticas es difícil de alterar. La posición analítica que he privilegiado llama a considerar tanto las promesas como sus límites. Así, en vez de seguir repitiendo estas proclamaciones casi como una cuestión de fe que se debe seguir ciegamente en la práctica investigativa y de educación, se abre la posibilidad de considerar seriamente el papel de la educación matemática en el estado actual del mundo. 
Finalmente, queda abierta la pregunta de cómo estas ideas de la comunidad internacional en educación matemática se pueden recontextualizar para pensar las especificidades de Colombia y América Latina, y es una invitación a todos los interesados en la educación matemática. A qué narrativa nos adherimos, implícita o explícitamente, y cómo nuestro deseo por una mejor enseñanza y aprendizaje genera inevitablemente posibilidades para unos y el cierre de acceso para otros son los dilemas políticos y éticos a los que nos enfrentamos todos los días como maestros, formadores de maestros e investigadores. En ellos, nuestra labor como educadores matemáticos nunca puede ser neutral, sino siempre altamente política. Más que una recomendación acerca de qué camino es conveniente seguir, una revisión como esta traza el terreno para que discutamos en qué direcciones queremos comprometernos a seguir.

\section{Referencias}

Aguilar, M. S. y Zavaleta, J. G. M. (2012). On the links between mathematics education and democracy: A literature review. Pythagoras, 33(2), 5-19. DOI: 10.4102/pythagoras.v33i2.164.

Alrø, H.; Skovsmose, O. y Valero, P. (2008). Inter-viewing foregrounds: Students' motives for learning in a multicultural setting. En M. César y K. Kumpulainen (eds.). Social Interactions in Multicultural Settings (pp. 13-37). Rotterdam: Sense.

Araujo, J. (2009). Uma abordagem sócio-crítica da modelagem matemática: A perspectiva da educação matemática crítica. Alexandria. Revista de Educação em Ciência e Tecnologia, 2(2), 55-68.

Artigue, M. y Blomhøj, M. (2013). Conceptualizing inquiry-based education in mathematics. ZDM, 45(6), 797-810. DOI:10.1007/s11858-013-0506-6.

Atweh, B.; Graven, M.; Secada, W. y Valero, P. (eds.). (2011). Mapping equity and quality in mathematics education. Nueva York: Springer.

Baker, D. P.; Goesling, B. y LeTendre, G. K. (2002). Socioeconomic status, school quality, and national economic development: A cross-national analysis of the "Heyneman-Loxley Effect" on Mathematics and Science Achievement. Comparative Education Review, 46(3), 291-312.

Barbosa, J. C. (2006). Mathematical modelling in classroom: A socio-critical and discursive perspective. ZDM, 38(3), 293-301. DOI:10.1007/ bf02652812.

Barwell, R.; Clarkson, P.; Halai, A.; Kazima, M.; Moschkovich, J.; Planas, N.; Setati-Phakeng, M.; Valero, P. y Villavicencio Ubillús, M. (eds.). (2016). Mathematics education and language diversity: The 21st ICMI Study. Cham: Springer International. 
Bernstein, B. (1993). La construcción social del discurso pedagógico. Bogotá: El Griot.

Bishop, A. J. (2005). Aproximación sociocultural a la educación matemática (P. Perry, trad.). Cali: Universidad del Valle.

Bourdieu, P. y Passeron, J. C. (1990). Reproduction in education, society, and culture (1990 ed.). Londres: Sage.

Camelo, F.; Mancera, G.; Zambrano, J. y Romero, J. (2013). Reflexiones sobre las potencialidades y dificultades en la iniciación de prácticas sociocríticas de modelación matemática. En G. García, P. Valero, C. Salazar, G. Mancera, F. Camelo, y J. Romero (eds.). Procesos de inclusión/exclusión. Subjetividades en educación matemática (pp. 119145). Bogota: Fondo Editorial Universidad Pedagógica Nacional.

Chassot, A. (2003). A Ciência é masculina? É sim, senhora! San Leopoldo, Brazil: Unisinos.

Chronaki, A. (2008). Technoscience in the "body" of education: Knowledge and gender politics. En A. Chronaki (ed.). Mathematics, technologies, education. The gender perspective (pp. 7-27). Volos: Thessaly University Press.

Chronaki, A. (2011). Disrupting "development" as the quality/equity discourse: Cyborgs and subalterns in school technoscience. En B. Atweh, M. Graven, W. Secada y P. Valero (eds.). Mapping equity and quality in mathematics education (pp. 3-19). Dordrecht: Springer Netherlands.

Civil, M. (2007). Building on community knowledge: An avenue to equity in mathematics education. En N. Nassir y P. Cobb (eds.). Improving access to mathematics: Diversity and equity in the classroom (pp. 105-117). Nueva York: Teachers College Press.

Cobb, P. y Hodge, L. L. (2007). Culture, identity, and equity in the mathematics classroom. En N. Nassir y P. Cobb (eds.). Improving access to mathematics: Diversity and equity in the classroom (pp. 159-171). Nueva York: Teachers College Press.

Coleman, J. S.; Campbell, E. Q.; Hobson, C. J.; McPartland, F.; Mood, A. M.; Weinfeld, F. D. y York, R. L. (1966). Equality of educational opportunity. Washington, D. C.: National Center for Educational Statistics-US Department of Health, Education, and Welfare.

D'Ambrosio, U. (2006). Ethnomathematics. Link between traditions and modernity. Rotterdam: Sense.

D'Ambrosio, U. (2011). Uma síntese sociocultural da história da matemática. São Paulo: Proem.

Damarin, S. (1995). Gender and mathematics education: Some implications from feminist science. En B. Grevholm y G. Hanna (eds.). ICMI Study on gender and mathematics education (pp. 129-136). Lund (Sweden): Lund University Press. 
Damerow, P.; Dunkley, M.; Nebres, B. y Werry, B. (eds.). (1984). Mathematics for all. París: Unesco.

Darragh, L. (2016). Identity research in mathematics education. Educational Studies in Mathematics, 93(1), 19-33. DOI:10.1007/s10649-016-9696-5.

Dewey, J. (1916). Democracy and education: An introduction to the philosophy of education. Nueva York: Macmillan.

Diaz, J. D. (2014). Governing equality. European Education, 45(3), 35-50. DOI:10.2753/EUE1056-4934450303.

Doğan, O. y Haser, Ç. (2014). Neoliberal and nationalist discourses in Turkish elementary mathematics education. ZDM, 46(7), 1-11. DOI:10.1007/s11858-014-0605-z.

Fennema, E. (1995). Mathematics, gender and research. En B. Grevholm y G. Hanna (eds.). Gender and mathematics education (pp. 21-38). Lund (Sweden): Lund University Press.

Forgasz, H. y Rivera, F. (eds.). (2012). Towards equity in mathematics education: Gender, culture, and diversity. Nueva York: Springer.

Foucault, M. (1973). The order of things. An archaeology of the human sciences. Nueva York: Vintage Books.

Frade, C.; Acioly-Régnier, N. y Jun, L. (2013). Beyond deficit models of learning mathematics: Socio-cultural directions for change and research. En M. A. Clements, A. J. Bishop, C. Keitel, J. Kilpatrick y F. K. S. Leung (eds.). Third international handbook of mathematics education (vol. 27, pp. 101-144). Nueva York: Springer.

Frankenstein, M. (1995). Equity in mathematics education: Class in the world outside the class. En E. Fennema y L. Adajian (eds.). New directions for equity in mathematics education (pp. 165-190). Cambridge: Cambridge University Press.

Furinghetti, F. y Giacardi, L. (2010). People, events, and documents of ICMI's first century. Actes d'història de la ciència $i$ de la tècnica. 3(2), 11-50. DOI:10.2436/20.2006.01.149.

García, G. (2003). Currículo y evaluación en matemáticas. Un estudio en tres décadas en la educación básica. Bogotá: Cooperativa Editorial Magisterio.

García, G.; Valero, P.; Salazar, C.; Mancera, G.; Camelo, F. y Romero, J. (2013). Procesos de inclusión/ exclusión. Subjetividades en educación matemática. Bogotá: Fondo Editorial Universidad Pedagógica Nacional.

Gerdes, P. (2007). Etnomatemática - Reflexões sobre Matemática e Diversidade Cultura. Ribeirão: Húmus.

Giacardi, L. y Scoth, R. (2014). Secondary school mathematics teaching from the early nineteenth century to the mid-twentieth century in Italy. En A. Karp y G. Schubring (eds.). Handbook on the history of mathematics education (pp. 201-228). Nueva York: Springer. 
Giménez, J.; Díez-Palomar, J. y Civil, M. (eds.). (2007). Educación matemática y exclusión. Barcelona: Graó.

Gutiérrez, R. (2013). The sociopolitical turn in mathematics education. Journal for Resarch in Mathematics Education, 44(1), 37-68.

Gutstein, E. (2006). Reading and writing the world with mathematics: Toward a pedagogy for social justice. NuevaYork: Routledge.

Halai, A.; Muzaffar, I. y Valero, P. (2016). Research rationalities and the construction of the deficient multilingual mathematics learner. En R. Barwell, P. Clarkson, A. Halai, M. Kazima, J. Moschkovich, N. Planas, M. Setati-Phakeng, P. Valero y M. Villavicencio Ubillús (eds.). Mathematics education and language diversity: The 21st ICM Study (pp. 279-295). Cham: Springer International.

Harding, S. (1998). Is science multicultural? Colonialisms, feminisms, and epistemologies. Bloomington (EEUU): Indiana University Press.

Healy, L., y Powell, A. (2013). Understanding and overcoming "disadvantage" in learning mathematics. En M. A. Clements, A. J. Bishop, C. Keitel, J. Kilpatrick y F. K. S. Leung (eds.). Third international handbook of mathematics education (vol. 27, pp. 69-100). New York: Springer.

Heyneman, S. P. y Loxley, W. A. (1982). Influences on academic achievement across high and low income countries: A re-analysis of IEA data. Sociology of Education, 55(1), 13-21.

International Association for the Evaluation of Educational Achievement. (2016). Brief history of IEA: 50 years of educational research. Recuperado de http://www.iea.nl/brief_history.html.

Jablonka, E. (2009). Mathematics for all. Why? What? When? En C. Winsløw (ed.), Nordic research in mathematics education. Proceedings from Norma08 (pp. 293-305). Rotterdam: Sense.

Jablonka, E. (2016). Mathematics education as a matter of achievement. En M. A. Peters (ed.). Encyclopedia of Educational Philosophy and Theory (pp. 1-6). Singapore: Springer.

Jablonka, E. y Gellert, U. (2011). Equity concerns about mathematical modelling. En B. Atweh, M. Graven, W. Secada, y P. Valero (eds.). Mapping equity and quality in mathematics education (pp. 223-236). Nueva York: Springer.

Jablonka, E., Wagner, D., y Walshaw, M. (2013). Theories for studying social, political and cultural dimensions of mathematics education. En M. A. Clements, A. J. Bishop, C. Keitel, J. Kilpatrick, y F. K. S. Leung (eds.). Third international handbook of mathematics education (vol. 27, pp. 41-67). Nueva York: Springer.

Jorgensen, R.; Gates, P. y Roper, V. (2014). Structural exclusion through school mathematics: Using Bourdieu to understand mathematics as a social practice. Educational Studies in Mathematics, 87(2), 221 239. DOI:10.1007/s10649-013-9468-4. 
Jurdak, M. E.; Vithal, R.; Freitas, E.; Gates, P. y Kollosche, D. (2016). Social and political dimensions of mathematics education. Nueva York: Springer.

Karp, A. y Schubring, G. (eds.). (2014). Handbook on the history of mathematics education. Nueva York: Springer.

Keitel, C. (2013). Introduction to section A: Social, political and cultural dimensions in mathematics education. En M. A. Clements, A. J. Bishop, C. Keitel, J. Kilpatrick, y F. K. S. Leung (eds.). Third international handbook of mathematics education (vol. 27, pp. 1-6). Nueva York: Springer.

Knijnik, G. (2012). Differentially positioned language games: Ethnomathematics from a philosophical perspective. Educational Studies in Mathematics, 80(1), 87-100. DOI:10.1007/s10649-012-9396-8.

Laisant, C.-A., y Fehr, H. (1899). Préface. L' Enseignement Mathématique, 1(1), 1-5.

Lange, T. (2016). Futures at stake: Children's identity work in the force field of social valorization of school mathematics. En P. Ernest, B. Sriraman y N. Ernest (eds.). Critical mathematics education. Theory, praxis and reality. Charlotte, NC-EEUU: Information Age.

Lerman, S. (2000). The social turn in mathematics education research. En J. Boaler (ed.), Multiple perspectives on mathematics teaching and learning (pp. 19-44). Westport (EEUu): Ablex.

Lerman, S. (2006). Cultural psychology, anthropology and sociology: The developing 'strong' social turn. En J. Maasz y W. Schloeglmann (eds.). New mathematics education research and practice (pp. 171188). Rotterdam: Sense.

Lindblad, S., Petersson, P., y Popkewitz, T. S. (2015). International comparisons of school results - A systematic review of research on large scale assessments in education. Stockholm: Swedish Reseach Council.

Llewellyn, A. (2015). Problematising the pursuit of progress in mathematics education. Educational Studies in Mathematics. 92(3), 299-314. DOI:10.1007/s10649-015-9645-8.

Martin, D. B. (2011). What does quality mean in the context of white institutional space? En B. Atweh, M. Graven, W. Secada y P. Valero (eds.). Mapping equity and quality in mathematics education (pp. 437-450). NuevaYork: Springer.

Meaney, T.; Edmonds-Wathen, C.; McMurchy-Pilkington, C. y Trinick, T. (2016). Distribution, recognition and representation: Mathematics education and indigenous students. En K. Makar, S. Dole, J. Visnovska, M. Goos, A. Bennison y K. Fry (eds.). Research in Mathematics Education in Australasia 2012-2015 (pp. 143-164). Singapore: Springer.

Meyer, J. W.; Ramirez, F. O. y Soysal, Y. N. (1992). World expansion of mass education, 1870-1980. Sociology of Education, 65(2), 128-149.

Morgan, C. (2014). Understanding practices in mathematics education: structure and text. Educational Studies in Mathematics, 87(2), 129143. DOI:10.1007/s10649-013-9482-6. 
Niss, M.; Blum, W. y Galbraith, P. (2007). Introduction. En W. Blum, P. L. Galbraith, H.-W. Henn y M. Niss (eds.). Modelling and Applications in Mathematics Education: The 14th Icm Study (pp. 3-32). Boston, MA: Springer US.

Norén, E. (2015). Agency and positioning in a multilingual mathematics classroom. Educational Studies in Mathematics, 89(2), 167-184. DOI:10.1007/s10649-015-9603-5.

Organisation for Economic Cooperation and Development OECD. (2014). PISA 2012 Results in Focus. What 15-year-olds know and what they can do with what they know. Recuperado de Paris: http://www.oecd. org/pisa/keyfindings/pisa-2012-results.htm.

Pais, A. y Valero, P. (2012). Researching research: mathematics education in the Political. Educational Studies in Mathematics, 80(1), 9-24. DOI:10.1007/s10649-012-9399-5.

Parra, A. (2017). Ethnomathematical barters. En H. Stahler-Pol, N. Bohmann y A. Pais (eds.). The disorder of mathematics education. Challenging the socio-political dimensions of research (pp. 89-105). Nueva York: Springer.

Planas, N. y López, P. (2007). El valor del contexto de los problemas para la inclusión de los alumnos inmigrantes recién Ilegados a la EsO. En J. Giménez, J. Díez-Palomar y M. Civil (eds.). Educación matemática y exclusión (pp. 179-189). Barcelona: Graó.

Planas, N. y Valero, P. (2016). Tracing the socio-cultural-political axis in understanding mathematics education. En A. Gutiérrez, G. C. Leder y P. Boero (eds.). The second handbook of research on the psychology of mathematics education. The Journey Continues (pp. 447-479). Rotterdam: Sense.

Popkewitz, T. S. (2004). The alchemy of the mathematics curriculum: Inscriptions and the fabrication of the child. American Educational Research Journal, 41(1), 3-34.

Postlethwaite, T. N. (1967). School organization and student achievement: A study based on achievement in mathematics in twelve countries. Estocolmo: Almqvist y Wiksell.

Radford, L. (2008a). Culture and cognition: Towards and anthropology of mathematical thinking. En L. D. English y M. G. Bartolini Bussi (eds.). Handbook of international research in mathematics education (2. ${ }^{a}$ ed., pp. 439-464). Nueva York: Routledge.

Radford, L. (2008b). The ethics of being and knowing: Towards a cultural theory of learning. En L. Radford, G. Schubring y F. Seeger (eds.). Semiotics in mathematics education: Epistemology, history, classroom, and culture (pp. 215-234). Rotterdam: Sense.

Rindermann, H. y Ceci, S. J. (2009). Educational policy and country outcomes in international cognitive competence studies. Perspectives on Psychological Science, 4(6), 551-568. Dol:10.1111/j.17456924.2009.01165.x. 
Schmidt, W. H., Cogan, L. S. y McKnight, C. C. (2010). Equality of educational opportunity. Myth or reality in us schooling? American Educator, Winter 2010-2011, 12-19.

Schoenfeld, A. H. (2004). The Math Wars. Educational Policy, 18(1), $253-$ 286. DOI:10.1177/0895904803260042.

Schreiber, C. (2014). Curricula and the making of the citizens. Trajectories from 19th and 20th century Luxembourg (tesis de doctorado), University of Luxembourg, Luxembourg. (PhD-FLSHASE-2014-17).

Secada, W.; Cueto, S. y Andrade, F. (2003). Opportunity to learn mathematics among Aymara-, Quechua-, and Spanish-speaking rural and urban fourth- and fifth-graders in Puno, Peru. En L. Burton (ed.). Which way social justice in mathematics education? (pp. 103-132). Westport, Conn.: Praeger.

Secada, W. (2011). The theoretical landscape. En B. Atweh, M. Graven, W. Secada y P. Valero (eds.). Mapping equity and quality in mathematics education (pp. 123-127). Nueva York: Springer.

Sfard, A. y Prusak, A. (2006). Telling identities: In search of an analytic tool for investigating learning as a culturally shaped activity. Educational Researcher, 34(4), 14-22.

Skovsmose, O. (1999). Hacia una filosofía de la educación matemática crítica (P. Valero, trad.). Bogotá: Una Empresa Docente.

Skovsmose, O. y Valero, P. (2012). Rompimiento de la neutralidad política: el compromiso crítico de la educación matemática con la democracia. En P. Valero y O. Skovsmose (eds.). Educación matemática crítica: Una visión socio-política del aprendizaje y enseñanza de las matemáticas (pp. 25-61). Bogotá: Ediciones Uniandes.

Stentoft, D. y Valero, P. (2009). Identidades-en-acción: Sobre la fragilidad del discurso y la identidad en el aula de matemáticas. Educação Unisinos, 13(2), 97-109.

Stillman, G. A.; Blum, W. y Biembengut, M. S. (eds.). (2015). Mathematical modelling in education research and practice: Cultural, social and cognitive influences. Cham: Springer International.

Tröhler, D. (2013). Los lenguajes de la educación. Los legados protestantes en la pedagogización del mundo, las identidades nacionales y las aspiraciones globales. Barcelona: Octaedro.

Tröhler, D. (2015). La medicalización de la investigación educativa actual y sus efectos en las políticas y reformas escolares. Profesorado. Revista de currículum y formación del profesorado, 19(1), 368-383.

Valero, P. (1999). Deliberative mathematics education for social democratization in Latin America. Zentralblatt für Didaktik der Mathematik, 99(1), 20-26.

Valero, P. (2008). Discourses of power in mathematics education research: Concepts and possibilities for action. PNA. Revista de investigación en didáctica de la matemática, 2(2), 43-60. 
Valero, P. (2012a). La educación matemática como una red de prácticas sociales. En P. Valero y O. Skovsmose (eds.). Educación matemática crítica: Una visión socio-política del aprendizaje y enseñanza de las matemáticas (pp. 299-226). Bogotá: Ediciones Uniandes.

Valero, P. (2012b). Perspectivas sociopolíticas en la educación matemática. En P. Valero y O. Skovsmose (eds.). Educación matemática crítica: Una visión socio-política del aprendizaje y enseñanza de las matemáticas (pp. 195-216). Bogotá: Ediciones Uniandes.

Valero, P. (2013). Mathematics for all and the promise of a bright future. En B. Ubuz, Ç. Haser y M. A. Mariotti (eds.). Proceedings of the 8th Congress of European Research in Mathematics Education (CERME 8) (pp. 1804-1814). Antalya, Turkey: Middle East Technical UniversityEuropean Society for Research in Mathematics Education.

Valero, P. (2017). Mathematics for all, economic growth, and the making of the citizen-worker. En T. S. Popkewitz, J. Diaz y C. Kirchgasler (eds.). A political sociology of educational knowledge: Studies of exclusions and difference (pp. 117-132). Nueva York: Routledge.

Valero, P. y García, G. (2014). Matemáticas escolares y el gobierno del sujeto moderno. Bolema, 28(49), 491-515. http://dx.doi.org/10.1590/1980-4415v28n49a02.

Valero, P.; Graven, M.; Jurdak, M.; Martin, D.; Meaney, T. y Penteado, M. (2015). Socioeconomic influence on mathematical achievement: What is visible and what is neglected. En S. J. Cho (ed.). The Proceedings of the 12th International Congress on Mathematical Education (pp. 285-301). Springer International.

Valero, P. y Meaney, T. (2014). Trends in researching the socioeconomic influences on mathematical achievement. ZDM, 1-10. DOI:10.1007/ s11858-014-0638-3.

Valoyez-Chavez, L. (2014). Colombian teachers' expectations of black and poor students' ability to learn algebra (tesis de doctorado). University of Misuri- Columbia. Recuperado de https://hdl.handle. net/10355/44224.

Valoyez-Chavez, L. y Martin, D. B. (2016). Exploring racism inside and outside the mathematics classroom in two different contexts: CoIombia and USA. Intercultural Education, 27(4), 363-379. DOI:http:// dx.doi.org/10.1080/14675986.2015.1106135.

Vasco, C. (2011, primer semestre). La presencia de Piaget en la educación colombiana, 1960-2010. Revista Colombiana de Educación, 60, 15-40.

Wade, P. (2010). The meaning of 'race' and 'ethnicity'. En P. Wade (ed.). Race and Ethnicity in Latin America (pp. 4-23). Pluto Books. 